\title{
PHÉNOMÉNOLOGIE DE L'EXPÉRIENCE SONORE
}

Pierre Kerszberg*

SINTESE - De acordo com a constatação de Nietzche, segundo a qual o destino da filosofia está ligado ao da música, devemos nos perguntar o que a fenomenologia traz para a compreensão do fenômeno sonoro. Trata-se de desenvolver a fenomenologia da consciência intima do tempo, levando-se em conta a riqueza do material musical. Este material se organiza em tomo de noçōes, aparentemente independentes umas das outras, mas de fato essencialmente ligadas, de ritmo e de surpresa. 0 conceito central que emerge desta pesquisa não é mais o de sentimento (ainda presente em Nietzsche, por intermédio de Schopenhauer), mas de atenção. Husserl limitava este conceito ao domínio da modificação imaginadora da experiência vivida.

PALAVRAS-CHAVE - Atenção. Consciência. Fenomenologia da música. Sentimento..
ABSTRACT - A la suite du constat de Nietzsche, suivant lequel le destin de la philosophie est lié à celui de la musique, il faut se demander ce que la phénoménologie apporte à l'intelligibilité du phénomène sonore. Il s'agit de développer la phénoménologie de la conscience intime du temps, en tenant compte de la richesse du matériau musical. Ce matériau s'organise autour des notions, en apparence indépendantes l'une de l'autre mais en fait essentiellement liées, de rythme et de surprise. Le concept central qui émerge de cette recherche n'est plus celui de sentiment (encore présent chez Nietzsche, par l'intermédiaire de Schopenhauer), mais d'attention. Husserl limitait ce concept au domaine de la modification imageante de l'expérience vécue.

MOTS-CLEFS - Attention. Conscience. Phénoménologie de la musique. Sentiment.

Peut-être une des manières de comprendre la nouvelle intelligibilité du monde annoncée par la philosophie contemporaine est-elle résumée dans cette phrase de Heidegger, extraite de la section 56 d'Etre et Temps: "la conscience morale parle uniquement et constamment sur le mode du silence". A cette formule on pourra opposer celle de Kant, qui évoquait dans la Critique de la Raison Pratique la façon dont la loi morale nous parle: c'est une "voix céleste". Devenue silencieuse, la loi morale trouve-t-elle son essence dans ce simple arrêt de l'émission vocale?

Université de Toulouse.

\begin{tabular}{|l|l|l|l|l|l|}
\hline VERITAS & Porto Alegre & v. 45 & n. 1 & Março 2000 & p. 103-116 \\
\hline
\end{tabular}


Une voix céleste pourrait être un son éminemment musical, comme dans la cosmologie pythagoricienne où les relations musicales de tonalité entre les corps célestes feraient ressembler notre système solaire à une lyre à sept cordes. Mais nous savons d'après Pythagore que s'il existe une harmonie musicale des corps célestes, elle est inaudible au commun des mortels; nous ne l'entendons pas parce qu'elle nous assourdit depuis notre naissance. A l'appui de cette thèse, au dire d'Aristote, les Pythagoriciens étaient forcés de faire une comparaison avec l'accoutumance au bruit qui finit par se résoudre en silence (ainsi le monde des forgerons). ${ }^{1}$ Le son musical le plus éminent devenait ainsi le prototype de l'habitus, condition paradoxale de tous les autres, puisqu'il fallait le considérer comme une habitude contractée dès notre naissance. Au lieu de chercher à lever le paradoxe en approfondissant la nature du son, Aristote a rejeté la thèse pythagoricienne en bloc en la détournant de sa vocation musicale. Pour des raisons cosmologiques, il se rabat en effet sur l'expérience du bruit: comme le bruit du tonnerre fend les pierres, les bruits excessifs des corps célestes devraient mettre notre terre en pièces. Point de bruit, donc point de musique.

Or, à l'époque moderne, le destin de la musique comme art, comme production spécifiquement humaine, a suivi un parcours étrangement similaire. Après avoir été exaltée par les romantiques comme moyen absolument autonome d'expression, la musique a vite subi de la part des philosophes une éclipse qui ne va pas sans rappeler la pure et simple évacuation de l'assourdissement évoqué par Aristote. Cette fois-ci, c'est une ontologie, non une cosmologie, qui fait oeuvre de destruction. Heidegger suit Hegel sur ce point: depuis le début du dix-neuvième siècle, la musique aurait trouvé son terrain de prédilection dans une attitude centrée sur le jeu des sentiments avec eux-mêmes, le jeu stérile de l'autocomtemplation des moindres soubresauts de notre âme livrée à elle-même. Lorsque la musique prétend dominer le monde esthétique, comme ce serait le cas avec la musique dite "absolue" (purement instrumentale) qui se développe vers la fin du dix-huitième siècle, elle met les autres arts sous sa coupe, mais elle y arrive seulement parce qu'elle se complait dans l'état purement affectif. Abandonné à soi dans l'univers musical, le sentiment, dit Heidegger dans son livre sur Nietzsche, dénote sa propre barbarisation grandissante. ${ }^{2}$ Parce que les formes esthétiques des époques antérieures étaient encore dirigées vers un sens de la vérité de l'être qui n'était pas exclusivement réservé aux misérables vibrations du moi, l'évolution de la musique indiquerait d'une manière particulièrement symptomatique ce que Hegel désignait déjà par la fin de l'art.

Si nous n'entendons plus la musique céleste des Pythagoriciens, s'il n'y a proprement plus rien à entendre dans la musique instrumentale des modemes, peut-être l'habitude contractée dès notre naissance fournit-elle au moins une

Aristote, Du Clel, 290b13-291a25.

M.Heidegger, Nietzsche, trad. P.Klossowski, Paris, Gallimard, 1971, vol.1, pp.85-86. 
tonalité fondamentale à notre être-au-monde? C'est bien ce que semble insinuer Heidegger. La loi morale se manifeste sous la forme d'un appel, et cet appel a la particularité de provenir de moi tout en me tombant dessus à l'improviste ("der Ruf kommt aus mir und doch über mich"), et c'est pourquoi l'appel, pour être authentique, se fait sans mot dire et se déploie dans le silence. Toutefois, si l'affectivité élémentaire ouvre bien par ses tonalités plus largement au monde que toute perception et que tout concept, si le sentiment comme Stimmung a une portée bien plus large et plus radicale que la rationalité, il faut que cette totalité ouverte nous tombe dessus elle aussi. De fait, Heidegger pense la Stimmung comme une musique complète par elle-même, comme la tonalité, l'accord qui définit la cohésion profonde de l'être-au-monde: "Une Stimmung est un air, écrit Heidegger dans un cours de 1929-30, au sens d'une mélodie, qui ne flotte pas audessus de la présence de fait, prétendue véritable, de l'homme, mais donne pour cet être le ton, c'est-à-dire harmonise (stimmt) et détermine (bestimmt) son style et sa manière d'être". ${ }^{3}$ Le son vient à l'auditeur; contrairement à la vision, la possession à distance est impossible: un caractère fondamental de la facticité du son est l'indépendance de l'effet sonore vis-à-vis des circonstances de sa production, comme si donc il n'était pas nécessaire de se soucier de sa raison d'être. D'où la possibilité de confusion du silence (c'est-à-dire l'appel qui appelle sans élever la voix) avec le fait que le son, pour une oreille préoccupée, apparaît le plus souvent comme coupé de sa source.

Or, si cette source pouvait encore subsister comme un aspect éminent de la conscience subjective, si la conscience était capable de constituer la musique qu'elle entend au lieu de se fier à la manifestation de l'être à travers la musique toute faite de la Stimmung, alors le poignant constat de Nietzsche sur notre tradition philosophique depuis l'Antiquité pourrait enfin avoir l'impact qu'il mérite: dans le royaume glacé des Idées, écrivait-il dans la section 372 du Gai Savoir, on ne pouvait que se mettre du coton dans les oreilles pour que la philosophie arrive à ses fins. Il y va ici du destin conjoint de la musique et de la philosophie. Comme l'a bien vu Nietzsche, la fin annoncée de la grande métaphysique va justement de pair avec une question essentielle sur le sens de la musique. D'abord il fait jouer le sens de la douleur tragique dans la musique de Bizet contre l'exaspérante répétition qu'il dénonce chez Wagner: Bizet réussit "sans le mensonge du grand style". Mais en dernière analyse, se demande-t-il dans l'une des dernières pensées de La Volonté de Puissance, "le concept de grand style serait-il en contradiction avec l'esprit de la musique?". 5 Si la musique était une erreur depuis le début des temps, si la grandeur qu'elle s'octroie traditionnellement n'était

Cité par M.Haar, Le Chant de la Terre, Paris, Editions de l'Herne, 1985, pp.92-93.

F.Nietzsche, Le Cas Wagner, trad. J.C.Hémery, ๔euvres Philosophiques Complètes vol.8, Paris, Gallimard, 1974, pp.21-22.

5 F.Nietzsche, La Volonté de Puissance, Paris, Gallimard, vol.2, p.338. 
qu'usurpation et mensonge, le déclin de la musique enlèverait à l'esprit tout espoir de se restituer à soi-même; n'entraînerait-il pas aussi avec lui, non le renouvellement de l'ontologie, mais la pure et simple paralysie philosophique?

Toute musique ne trahit pas l'essence de la musique: telle est la position fondamentale de Nietzsche, qu'il a tenté de justifier dès l'ouvrage de jeunesse qu'est La Naissance de la Tragédie. Nietzsche y fait l'hypothèse que toute musique dérive du désir de danser ensemble dans une communauté qui nous rassemble; elle est un moyen pour annuler la séparation entre les individus d'une communauté. La danse n'est-elle pas cette activité sociale qui se rapproche le plus d'une réponse esthétique idéale - une manière d'être ensemble où le présent est entièrement absorbé, où l'intérêt est saturé? Inséparable de la danse, l'essence de la musique est donc aussi inséparable d'un jeu de figures, et cette inséparabilité se trouverait exprimée au plus haut degré dans le drame musical grec. Or, ce même drame semble également prouver l'impossibilité que la musique soit jamais intelligible d'un point de vue phénoménologique. En effet, une fois portée sur la scène, la figure pourrait être bougée de la manière la plus visible, on pourrait l'animer et l'illuminer de l'intérieur: toutes ces variations ne changeront rien au fait que la figure restera un simple phénomène, "d'où nul pont ne mène à la réalité vraie, au coeur lui-même du monde". Et Nietzsche d'ajouter: "C'est à partir de ce coeur, en revanche, que parle la musique" ${ }^{\text {. }}$ La musique partirait directement de ce coeur, sans que le raccord avec le phénomène soit jamais réalisé. Mais ce raccord est-il même pensable? La musique ne fait justement que parler à partir de son fond: ce n'est pas encore pour elle une manière de se faire entendre. Le phénomène intervient alors comme "le baume salutaire d'une illusion délicieuse" :' la force apollinienne rétablit certes l'individu (i.e., le phénomène), mais comme une illusion, tandis que le fond dionysiaque reste inaudible. Prise entre l'illusoire et l'inaudible, la musique - la nôtre, celle que nous composons avec la force de notre propre imagination - "symbolise une sphère antérieure et supérieure à toute manifestation", l'Un originaire (ou la volonté aveugle au sens de Schopenhauer), et il faut donc se demander: qu'est-ce qu'on entend quand on entend un symbole? Est-ce l'Un originaire qui se fait entendre, et si tel est le cas, comment pourrions-nous le savoir? Symbole s'entend ici au sens de Schopenhauer: reproduction immédiate du vouloir et sa réalité supérieure, au contraire de l'image qui, en tant que réalité médiate, est seconde par rapport au phénomène.

Cette esquive du phénomène rappelle les deux types de connaissance que Schopenhauer lie au double rapport que chacun entretient avec son propre corps ${ }^{8}$ :

F.Nietzsche, La Naissance de la Tragédie, \$21, trad. Ph.Lacoue-Labarthe, Paris, 1977, p.127.

Ibid., §21, p.124.

A.Schopenhauer, Le Monde comme Volonté et comme Représentation, trad. A.Burdeau, Paris,

Presses Universitaires de France, 1966, pp.141sq. 
d'une part ce corps m'est connu comme objet, c'est-à-dire médiatement, au travers d'une représentation; d'autre part il m'est connu dans le sentiment par lequel je l'éprouve immédiatement comme moi-même, donc comme sujet, dans l'action volontaire. C'est précisément le sentiment dont je voudrais avoir un concept, mais cela est impossible. En ce qui conceme la musique, le concept est inutile: le sens de la musique est contenu dans le langage que la raison ne comprend pas, et ce langage est celui du sentiment. Pourtant, dans des textes et fragments annexes à La Naissance de la Tragédie (en particulier dans le texte intitulé "La vision dionysiaque du monde"), Nietzsche va plus loin que Schopenhauer et s'efforce de penser le phénomène musical en tant que phénomène et rien que phénomène. Ce dernier est-il un monde à part de la raison et du sentiment, ou bien est-il une sorte de somme des deux? Pour arriver à le comprendre, il faut commencer par retourner à un sens plus naïf de symbole: image imparfaite, signe conventionnel de suggestion sur lequel il faut se mettre d'accord. Moins élevé ontologiquement parlant, ce sens de symbole est aussi plus proche de l'apparence. Telle est bien cette recherche d'un "nouveau monde symbolique" que Nietzsche annonçait dès le début. ${ }^{9}$ il s'agit de penser rien moins qu'un nouveau double rapport au corps, non plus interne/externe, mais partiel/total.

Il existe certains états inconscients de la volonté, où commence à poindre la volonté qu'a la volonté de venir à la rencontre du phénomène dont elle constitue le fondement. Ces états constituent le sentiment au sens de Schopenhauer. Or, lorsque l'homme est porté au plus haut degré de ses facultés symboliques, i.e. dans le dithyrambe dionysiaque, ce qui tente de s'extérioriser, c'est "quelque chose de jamais ressenti". ${ }^{10}$ Dans la profondeur du sentiment se trouvent des moyens symboliques de communication avec l'inconscient qui sont eux-mêmes inconscients, qui sont donc les plus fidèles à l'Un originaire: le langage des gestes et celui des sons. Quel est leur rapport au phénomène? II s'agit soit d'un accompagnement (par le geste qui symbolise le sentiment), soit de "l'ensemble des gestes" où s'annonce justement le nouveau monde symbolique. Car dans cet ensemble le son devient symbole des mouvements de la volonté - plaisir ou déplaisir - mais "sans la moindre représentation d'accompagnement". ${ }^{11}$ Alors que chaque geste pris séparément s'en tient toujours à l'ordre de la représentation, leur ensemble nous propulserait dans l'ordre de l'être. Comment cela se peut-il? On passe du partiel au total, non par amplification du partiel, mais par intermittence du partiel. Le geste n'est plus un accompagnement, mais une plénitude claire par soi: "Par exemple, quand nous parlons de la frayeur soudaine, de la manière dont la douleur 'frappe, tire, élance, pique, coupe, mord, chatouille'.

\footnotetext{
La Naissance de la Tragédie, §2, p.35.

Ibid., p.35.

"La vision dionysiaque du monde", p.307.
} 
Par là semblent être exprimées certaines 'formes d'intermittence' de la volonté, bref - dans une symbolique de la langue des sons - la rythmique". Un des arguments les plus retentissants justifiant la rupture ultérieure avec Wagner, ce sera précisément que la musique ne résulte pas d'une amplification du geste, de la suggestion ou de tout ce qui relève du psychologique.

Or, une des tâches fondamentales de la phénoménologie de la conscience intime du temps, telle qu'elle a été définie par Husserl dans les Leçons pour une Phénoménologie de la Conscience Intime du Temps de 1905, était de comprendre comme la forme originaire du rythme - une succession de perceptions - pouvait devenir une perception de succession, c'est-à-dire comment l'intermittence ellemême s'intègre dans le continuum du temps. Comment la conscience actuelle estelle capable de retenir le passé comme passé? Comment la partie déjà écoulée d'un objet temporel est-elle préservée dans le moment actuel? Si la conscience était incapable de le faire, au son d'une mélodie, entendu maintenant, s'adjoindrait l'expérience d'un chaos tonal - quelque chose de tout juste passé ne pourrait jamais faire partie du présent sans rompre avec lui. La solution est de concevoir des phases de "tout juste passé" (appelées phases de rétention) modifiées à chaque nouvelle appréhension. Prises ensemble, ces modifications définissent un continuum de gradations qui est constamment modifié. Or, comme les appréhensions passent continuellement l'une en l'autre derrière le maintenant, elles contribuent toutes à une appréhension de ce maintenant qui n'est plus finalement, et paradoxalement, qu'une limite idéale. ${ }^{12}$ Aucun moment du contenu tonal n'est disponible dans le souvenir de ce qui est juste passé, il ne contient que la conscience de ce son en tant que passé, et rien de plus que cette conscience.

L'apparente évaporation du contenu tonal dans le flux temporel absolu de la subjectivité n'est sans doute pas étrangère à la navrante simplicité du phénomène décrit par Husserl: un son continu, une mélodie simple. Néanmoins, Husserl a souvent insisté sur le fait que la vision qui donne l'essence est à la fois originaire et adéquate dans le cas du son, alors qu'elle n'est pas adéquate à son objet dans le cas de l'objet perçu dans l'espace. A la différence de l'oeil qui nous porte vers des significations déchiffrables à même la surface du visible, l'oreille semble inerte, elle parait se contenter de sa plénitude. Le sens qui affleure par l'intermédiaire de l'oeil est accompagné de mouvements comme ceux des cils et des lèvres, qui tous contribuent à véhiculer ce sens, tandis que l'oreille ne révèle pas un sujet capable de prendre conscience de soi en s'écoutant. C'est pourquoi l'objet spatial se montre au travers d'esquisses qui le préfigurent, chaque esquisse foumissant l'occasion à l'intentionnalité de la conscience de constituer cet objet pour la conscience. Il y a bien un équivalent des esquisses dans le cas du son: ce sont les modulations, mais celles-ci s'offrent d'elles-mêmes comme déjà constituées, car il est bien connu que

${ }^{12}$ E.Husserl, Leçons pour une Phénoménologie de la Conscience Intime du Temps, §16, trad. H.Dussort, Paris, Presses Universitaires de France, 1964, p.57. 
même là où les sons sont reconnus comme étant incomplets, l'auditeur perçoit que l'incomplétude de l'un est compensée par celle de l'autre au moyen d'un processus de résolution. Le monde du son est-il encore compatible avec l'intentionnalité de la conscience? L'eidos visuel sert de tremplin pour amener la pensée au seuil de la mise hors circuit de ses croyances naïves et toutes faites: c'est le moment où l'oeil s'efforce d'arrêter son clignotement, quitte l'attitude naturelle, et va s'installer dans l'intentionnalité du pur voir - qui en principe coïncide avec l'essence de l'objet.. Or, si l'oreille est déjà absolument inerte et passive dans l'attitude naturelle, la situation de l'écoute jouit du privilège d'exécuter une mise hors circuit des validités naïves sans même forcer ou altérer son pouvoir. C'est pourquoi une "écoute réduite"13 n'est pas du tout une écoute, tout comme la musique dionysiaque est inaudible tant qu'elle ne se compromet pas avec l'illusion apollinienne.

Certes, il semble plus facile pour nous de rester insensible à ce que nous voyons qu'à ce que nous entendons; ce qui est vu reste à distance, tandis que ce qui est entendu ne s'arrête pas aux limites tracées par le corps. Mais lorsqu'il passe aux synthèses passives de la conscience - ce moment de la conscience qui précède la constitution active d'un objet par la conscience, c'est justement cette apparente insensibilité que Husserl nie. Il insiste sur la contradiction inhérente à toute perception spatiale: elle prétend en effet à l'impossible donnée en personne de l'objet perçu, puisqu'une perception qui donnerait l'objet sous tous les côtés est impensable. Impensable en effet est l'indétermination déterminée, l'horizon vide qui n'est pas néant, qui entoure ce qui apparaît en personne. Impensable mais non inaudible: l'intentionnalité est ici une réponse à une exigence ou un appel de l'objet mondain. Le perçu nous crie pour ainsi dire: "approche-toi plus près et toujours plus près, considère-moi et fixe-moi alors en changeant ta position, ton regard"14. Tout ce qui relève du regard vers l'eidos pur est conditionné par un appel, plus primordial que toute l'opération de constitution d'objet qui va suivre.

Bien entendu, cet appel est plus facile à comprendre dans le cas où l'objet en face de nous est notre interlocuteur. Dans le second chapitre de Matière et Mémoire, qui reste à ce jour le texte le plus pénétrant en vue d'une phénoménologie de l'écoute, Bergson imagine le cas où j'écoute quelqu'un qui me parle une langue inconnue de moi. ${ }^{15}$ Lorsque les sons sont perçus dans leur matérialité brute, mon corps leur ajoute un schème moteur, c'est-à-dire une forme qui dérive de la coordination entre les impressions auditives et les muscles de ma propre voix. Je me place directement au milieu des idées correspondantes; un discours intérieur de mon propre cru se met en marche, rendant de la sorte les

Au sens où en parle Pierre Schaeffer dans son Traité des Objets Musicaux, Paris, Le Seuil, 1966.

E.Husserl, De la Synthèse Passive, trad. B.Bégout et J.Kessier, Grenoble, J.Million, 1998, p.98.

H.Bergson, Matière et Mémoire, Paris, Presses Universitaires de France, 1959 (Edition du Centenaire), pp.261sq. 
images auditives distinctes. Mon discours intérieur commence à chevaucher le discours qui demande à être compris, ce qui produit l'ébauche d'une intelligibilité complète. Le schème moteur dans mon corps, dit Bergson, est comparable à un récipient vide qui détermine la forme de la matière qui s'y précipite. Les images sont devenues des idées quand l'impression que j'ai de tout mon corps est comme celle que j'ai de ma tête, c'est-à-dire une forme vide mais non inerte, permettant aux sons de résonner dans la cavité éprouvée comme creuse.

Que se passe-t-il dans le cas de l'écoute de la musique? Bergson dit que nous ne pouvons que répéter intérieurement les sons. ${ }^{16}$ le discours intérieur, c'est-à-dire l'ébauche d'une réponse à ce qui est entendu, subsiste maintenant par soi, puisque l'intelligibilité totale de ce que la musique nous présente n'est même pas imaginable (tandis que pour Schopenhauer, la musique reste parfaitement intelligible même si personne ne peut l'expliquer). L'idée d'une note qui a une certaine hauteur nous rappelle l'effort que le muscle tensile de nos cordes vocales devrait faire pour produire le son. Or, même si cet effort peut se propager à d'autres parties du corps - les pieds, les mains, etc. - il n'a même pas besoin d'arriver à un ensemble de gestes pour atteindre l'Etre de la musique - pas plus que l'Etre tout court: il suffit pour cela qu'il soit perçu immédiatement comme un acte indivisible, c'est-à-dire une succession qui ne peut être brisée comme les points d'une ligne. C'est dire que la hauteur du son appelle son dépassement dans la durée, et même une durée pure: une succession sans distinction, une représentation privée de tout symbole. Si le moi se rappelle bien les sons passés tandis que la mélodie continue, cela n'est possible que s'ils ont en quelque sorte fondu l'un en l'autre, au point de ne laisser aucune liberté à l'espace et l'effort intellectuel d'abstraction. Or, pouvons-nous demander, des sons qui fondent l'un en l'autre ne livrent-ils pas autre chose qu'une cacophonie? Le rythme n'est-il pas cet espace sonore qui, empêchant les sons de s'interpénétrer, les rend par là même plaisants et intéressants? Quelle est la trace laissée par les sons dans l'esprit de l'auditeur, tandis que ceux-ci suivent inexorablement leur progression dans le temps?

Il se fait que, indépendamment des puissances de l'écoute, les sons euxmêmes engendrent un résidu ou une trace: ils résonnent. Or, là où l'on attend de la phénoménologie qu'elle décrive l'expérience vécue de la résonance, Husserl dit que la résonance est l'un de ces faits de la nature qui doivent être mis entre parenthèses. Comme l'intuition du passé est une conscience originaire, la résonance appartient non à la rétention mais à la perception. ${ }^{17}$ Etrange dissymétrie dans l'argumentation husserlienne: si l'on accordera qu'un souvenir n'est pas une perception affaiblie, pourquoi l'écho serait-il simplement un son affaibli?

16 H.Bergson, Essai sur les Données Immédiates de la Conscience, Edition du Centenaire, p.26.

Leçons, §12, p.47. Voir les commentaires de Paul Ricoeur dans Temps et Récit, t.1, Paris, Le Seuil, pp.30-41. 
C'est à Saint Augustin, dans le livre XI des Confessions, que revient le mérite d'essayer de penser la résonance d'une manière phénoménologique; la remémoration d'un son qui a résonné ouvre précisément sur une dimension d'espace à l'intérieur de la séquence temporelle. Même s'il n'a rien de spatial en lui, le présent laisse derrière lui une sorte d'espace temporel (spatium temporis), ce qui permet de le mesurer maintenant comme s'étant produit dans le passé. Dans le passé il y avait une tension qui a fait contracter le temps en une sorte d'espace. Quel est le rôle de l'impression dans cette mise en marche de la tension? Augustin discute deux exemples très différents pour expliquer le mécanisme de cette tension. Premier exemple: je récite les mots d'un poème que je connais par coeur. Dans ce cas, ce ne sont pas les syllabes de différentes longueurs que je mesure, puisqu'elles sont déjà passées, mais leurs empreintes qui sont fixées dans la mémoire, telles qu'elles ont été déposées par la première impression (affectio). C'est le second exemple qui concerne la musique. Ici l'affectio n'est plus directement présente, comme lorsque je chante un chant que je connais déjà. $\mathrm{Au}$ début, l'attente est dirigée vers le chant dans sa totalité, plus tard la mémoire tend vers la portion du chant qui est déjà passée; grâce à l'attention (attentio), l'intention présente consiste en une transposition active de ce qui était futur dans ce qui est devenu passé. C'est pourquoi le temps est une distensio de l'âme, une distension recueillie par l'attention, où le maintenant ponctuel et évanescent est certes prolongé, mais dont la prolongation est combattue par l'intentio, qui est un effort visant à intensifier l'attente. Qu'est-ce qui, dans la distension, relève de la passivité d'un affect (impression)? Augustin et Husserl s'accordent finalement sur ce point: quelque chose demeure tout au long de la récitation ou du chant dans la mesure où poème et vers (et finalement tout discours) sont traversés en pensée.

On le voit: à cause d'une tension psychologique antérieure à l'acte de réciter ou de chanter, l'expérience quotidienne s'est désagrégé dès le début. Je me prépare activement à faire entendre quelque chose que je connais déjà. La préparation masque, ou en tout cas atténue considérablement, toute dimension de surprise dans l'écoute d'un son que je produis moi-même. Les différentes tensions qui s'affrontent dans la distension sont toutes des expressions d'une tension initiale ou premier affect, tension qui va diminuant pour revenir ensuite sous la forme d'une pensée qui s'affecte elle-même. Que serait une tension en réponse à l'inattendu, que le son soit produit par moi ou qu'il vienne de l'extérieur?

La musique moderne est un art de la surprise. L'inattendu est un élément structurel de la musique, comme on le voit dans la manière par laquelle elle se définit vis-à-vis d'un commencement. La musique dite "classique" abonde d'exemples qui montrent combien les frontières musicales sont hautement perméables, à tel point que le début d'une mélodie n'est jamais vraiment repérable vis-à-vis d'une phrase d'accompagnement. Dans un morceau comme le premier mouvement de la Neuvième Symphonie de Beethoven, on peut parler de deux commencements. Même s'il implique une certaine connaissance par anticipation, 
le second commencement n'est annoncé par rien; ici il n'y a pas d'attente au sens tonal. Ce moment est essentiellement une force de dislocation à l'intérieur d'un tout stable, une dislocation qui ajoute à cette stabilité au lieu de lui retirer quelque chose. C'est tout le monde quotidien qui, bien loin d'être englouti et dépassé par une force dionysiaque qui tout à coup se ferait entendre, se trouve déplacé et transposé. Que s'est-il passé entre les deux commencements? Chacun, en tant qu'impression originaire, peut être considéré comme un instant pur, dans la mesure où il se détache comme événement purement temporel de l'environnement de temps spatialisé où il est immergé. Mais tandis que l'instant pur du premier commencement n'est pas remarqué en tant que pur, mais seulement en tant que partie du temps spatialisé, celui du second commencement représente l'advenue du temps pur pour une conscience vivante: le temps proprement musical.

J'affirme que toute la phase entre les deux premiers commencements est un cas de durée pure: en elle, le changement est la chose même, c'est un changement où rien ne change (selon la définition de la durée pure de Bergson), puisque dans la conscience du sujet auditeur le rayon de l'attention est alors ré-orienté dans une direction qui n'est pas encore déterminée. Cela implique-t-il que le temps musical s'installe sur les ruines du temps objectif, révélant de la sorte une réalité plus profonde inaccessible au monde objectif? En fait, la durée pure entre les deux premiers commencements est même la seule instance de durée pure au sens de Bergson. II n'y a pas de durée pure qui ne soit elle-même en train de se faire dans les phases de double commencement. Une fois que l'attention spécifiquement musicale s'est mise en mouvement, elle est loin d'être indivise, car même là où elle se manifeste, ce n'est jamais qu'exceptionnellement, sans doute parce que la pression grandissante exercée sur notre corps ne peut dépasser un seuil relativement bas. C'est pourquoi l'aspect décisif de l'attention en musique pourra être caractérisé comme dérive attentionnelle. Quand j'écoute la musique, je peux regarder par la fenêtre et observer le passage des nuages, songer à un poème, me réjouir de voir un ami demain; donc rêver sans pourtant tomber aussi bas que la simple sensation de se laisser bercer - le bercement serait l'équivalent sensible d'une fusion réussie des sons. ${ }^{18}$ De temps à autre, mon attention se dirigera à nouveau vers la musique, pour ensuite se laisser aller à la dérive une fois de plus. Or, ces événements ne sont pas de simples accidents psychologiques: la perception vécue de la musique est une dérive au sens de Husserl, certes atténuée par le phénomène de l'intermittence: le passé immédiat est souvent modifié et réinterprété - non pas constamment comme ce serait le cas pour un son continu examiné par Husserl - en fonction de chacun des moments qui se présentent à la conscience comme deuxième commencement. Quant à la musique, elle dérive à sa propre

18 Contre l'argument bergsonien selon lequel l'essence de la musique est dans le bercement, voir G.Marcel, "Bergsonisme et Musique", La Revue Musicale, 6 (1925), p.219-229. 
manière grâce au système des modulations. Le fait fondamental est que ces deux dénives ne pourraient jamais coïncider ou se recouvrir parfaitement, ce qui justifie la forme d'intermittence propre à l'être qui se manifeste dans la musique. En effet, la durée pure n'est jamais donnée dans une pleine présence du présent, sauf quand elle est en train de se constituer comme une telle durée; l'aspect facticiel ou mondain de la musique est donc neutralisé pendant cette phase. Si la durée pure est bien le pont qui relie les deux premiers commencements, cela entraíne que le matériau sonore dans sa facticité ne se fait pas entendre d'emblée; graduellement la conscience attentionnelle se dirige spécifiquement vers lui, mais de telle sorte que la neutralisation du début n'est pas annulée une fois pour toutes. Au contraire, elle revient par intermittence dans le champ de conscience grâce aux phases de dérive attentionnelle: la durée pure est pour ainsi dire remémorée par procuration, lorsque le facticiel musical s'est déplacé sur un facticiel d'un autre ordre. La perception de la musique est l'écho d'une durée pure originelle qui n'a jamais été vécue dans un présent, non pas cette durée elle-même.

Dans le cas contraire, nous serions renvoyés à l'antinomie propre à l'art musical établie par Hegel dans son Esthétique: cet art a pour contenu le sentiment, alors même que le sentiment ne pourrait jamais constituer que l'enveloppe d'un contenu; plutôt qu'une forme d'intermittence, il y aurait alors quelque chose de fondamentalement évanescent dans la musique, puisque forme et contenu soit tiennent ensemble pour un temps, soit se perdent simultanément.

Que nous fassions l'expérience du son musical comme une anticipation paradoxale de la surprise, voilà qui rappelle le processus de constitution transcendantal d'un autre ego dans la Cinquième Méditation Cartésienne de Husserl. Qu'il s'agisse de Hegel, de Schopenhauer ou de Bergson, l'argument est souvent le même: une composition musicale est comme un tout organique associé à des sentiments définis, de sorte que saisir l'être de la musique pourrait équivaloir à constituer une autre subjectivité. Quand un autre individu entre dans mon champ perceptif, cet Autre est là en personne, en chair et en os. Or, puisque tout processus de constitution commence par une réduction transcendantale du donné aux actes de mon ego, cet Autre ne peut pas non plus être donné d'une manière originelle. Il faut que la surprise soit atténuée pour que l'expérience de l'altérité radicale soit possible, c'est-à-dire pour que l'Autre soit constitué dans l'expérience de l'intersubjectivité; et cette atténuation donne sens à la réduction phénoménologique comme expérience vécue. Je commence avec une réduction de la subjectivité étrangère à ma sphère transcendantale propre; je vis dans ce monde réduit, d'où ressort mon corps physique (Körper) comme mon corps propre (Leib). Je ne pourrais atteindre un autre ego par l'entremise de quelque raisonnement par analogie. Le corps de l'autre m'est apprésenté, induisant une modification de mon propre ego, qui est justement cette atténuation des privilèges de mon corps sur un autre corps. Par transfert aperceptif issu de mon corps propre, qui est un processus entièrement passif, le sens d'un ego est mis en 
rapport avec celui d'un autre ego; plus précisément: un sens est transposé dans l'autre, processus appelé appariement (Paarung). Husserl insiste néanmoins toujours sur le fait que l'expérience de l'étranger ne peut s'effectuer que par des apprésentations se déroulant de manière synthétique et concordante; le comportement de l'autre est une indication de l'autre seulement sur le mode de la confirmation positive; si ce n'était pas le cas, l'appariement ne fournirait même pas le support associatif pour que l'analogie entre l'ego et l'alter ego puisse commencer. Mais il n'existe pas de relation universelle déterminable entre un sentiment et une forme musicale. C'est pourquoi l'expérience sonore est une expérience de l'altérité qui nous tombe pour ainsi dire dessus, entraînant la possibilité du désaccord et de la discordance.

Que pourrait bien être une réduction qui ne conduit pas automatiquement à la résolution et à la concordance? Dans la dérive attentionnelle, il se produit un appariement tout à fait particulier entre les deux temps de l'expérience - le temps spatialisé et le temps de la durée pure. Le premier semble tout d'abord ployer sous la pression du second. Mais le temps spatialisé doit bien rester à l'arrière-plan durant la phase initiale, et inversement la durée pure reste à l'arrière-plan du temps spatialisé lorsque le vécu est en phase avec la musique selon sa dynamique propre. De plus, la modification attentionnelle vers ce qui est potentiellement signifiant en musique peut ne pas apparaître du tout - parfois en effet la musique m'ennuie. Mais si elle apparait, elle est elle-même le résultat d'une dérive attentionnelle qui prend du temps. Par l'intermédiaire de la dérive, l'attention passe par des phases de tension et de relâchement, mais sans jamais aller aussi loin qu'une force intentionnelle. A aucun moment la musique ne se présente comme une autre sphère propre. Les deux temps, durée pure et temps spatialisé, sont donc co-appréhendés tout au long de l'expérience musicale, en dépit du fossé d'altérité qui les séparent. L'interaction entre les deux temps dans la dérive attentionnelle est comme un jeu d'éclipses. Pendant la phase des deux premiers commencements, le temps spatialisé est complètement éclipsé par la durée pure; mais c'est aussi le temps où il ne se passe pratiquement rien de musical pour l'auditeur - même si bien entendu il se passe une foule de choses capitales du point du vue de la technique de composition. Après le deuxième commencement, un temps est toujours juxtaposé à l'autre, sans qu'il se produise jamais le moindre transfert complet de l'un à l'autre. Tout au plus viendront-ils en contact, mais ce contact n'ira jamais jusqu'à la fusion.

D'après le premier livre des Ideen de Husserl, l'attentionnalité dirigée vers l'objet reste un cas particulier de l'intentionnalité: les variations de l'attention n'altèrent pas le sens contenu dans le noyau noématique. ${ }^{19}$ Néanmoins le problème est que les mutations attentionnelles sont des transformations qui

19 E.Husserl, Idées Directrices pour une Phénoménologie, t.1, §92, trad. P.Ricoeur, Paris, Gallimard, 1950, pp.317-322. 
peuvent affecter l'ensemble du vécu - et cela d'une manière soudaine, comme lorsqu'au beau milieu de la perception d'un arbre nous toumons le regard vers un objet de souvenir qui nous passe par la tête. De plus, il y a une dissymétrie flagrante entre les deux fonctions: l'inattention, dit Husserl, serait le mode de la conscience morte, alors que l'intentionnalité vide reste une fonction de la conscience vivante. Ce qui importe, c'est que les modes de l'attention se distribuent selon des degrés et non des profils (comme dans le cas de la perception spatiale): tel moment de l'objet sera préféré à tel autre, tel moment sera remarqué à titre primaire au détriment d'un autre, etc.; autrement dit, ces modes révèlent bien des relations d'éclipse entre apparences noématiques, où ce qui est caché continue d'apparaitre sous la forme d'une rétention; à son tour, ce qui est ainsi retenu peut se prêter à une présentification sous la forme du ressouvenir. L'attentionnalité est donc la matrice de tous les types de modification de représentation. Mais cet enchaînement du vécu, jusqu'où peut-il se poursuivre? Husserl ne le pense que sur le mode de l'idéalité: il peut se poursuivre de la sorte à l'infini. D'où l'ambiguité du fameux exemple esthétique de la galerie de Dresde. ${ }^{20}$ un nom prononcé me fait songer à cette galerie, ma dernière visite de cette galerie, le tableau de Teniers qui représente lui-même une galerie de tableaux, ces tableaux représentent eux-mêmes des tableaux, et ainsi de suite. Qui pourrait croire que le noème de la perception initiale ne se dissout pas dans l'oubli au fur et à mesure que l'emboîtement se poursuit? L'intentionnalité n'est-elle pas un cas d'attention absolue, un vécu que personne ne pourrait jamais vivre en fait? Et une attention absolue, qui ne s'autorise aucune distraction, n'est-elle pas justement comparable à un état de mort? Certes, nous ne sommes ici qu'au niveau de la modification imageante, où les redoublements de l'image se poursuivent sans fin assignable. En principe, tout autre est la modification insigne qu'est l'opération de neutralisation (epokhè). ${ }^{21}$ mais par contraste avec la modification imageante, il est exclu, dit Husserl, qu'on puisse la répéter, ne fût-ce qu'une fois; c'est donc en fait une autre cas d'attention absolue, que Husserl caractérise comme une conscience traversée par une coupure radicale.

Le rayon attentionnel se donne comme irradiant du moi, comme un Ichstrahl. Par conséquent, si la dérive attentionnelle n'est pas un acte gratuit et arbitraire comme pourrait l'être une variation éidétique - alors il faut se représenter le sujet capable d'une telle dérive comme multiple. Or, un sujet multiple, n'est-ce pas une des conséquences de la phénoménologie de la conscience intime du temps? Une rétention présente doit elle-même faire partie du flux du temps, d'où la distinction entre l'intentionnalité transversale qui retient les objets et leur succession, et l'intentionnalité longitudinale où les rétentions elles-mêmes apparaissent à la conscience dans leur succession et leur durée. Cette seconde intentionnalité, qui 
est une réflexion de l'ego sur ses propres vécus, est problématique. Car si l'intentionnalité longitudinale était une modification de la première rétention transversale se produisant dans le temps, nous ne pourrions jamais saisir une rétention dans son identité. Un vécu serait constitué par une intentionnalité d'ordre supérieur chaque fois qu'il se présente à nous. Mais dans le monde sonore, cet obstacle se retourne en un bienfait: le sujet pourrait fort bien être multiple comme un ton fondamental est entouré de ses harmoniques; la dérive attentionnelle est le jeu organisé de ces harmoniques qui constituent un sujet. Le sujet qui réfléchit sur ses propres vécus les atténue au lieu de les modifier. Par là, au lieu de contempler une autre vie, il s'approche de sa propre mort tout en la retardant. 